\title{
Doubly forbidden second-harmonic generation from isotropic suspensions: Studies on the purple membrane of Halobacterium halobium
}

\author{
P. Allcock, D. L. Andrews, ${ }^{*}$ S. R. Meech, and A. J. Wigman \\ School of Chemical Sciences, University of East Anglia, Norwich NR4 7TJ, United Kingdom
}

(Received 28 August 1995)

\begin{abstract}
In this paper we report results on second-harmonic generation (SHG) from aqueous suspensions of the purple membrane of Halobacterium halobium. A detailed theory describing this SHG signal is supported by results for the angular distribution of the harmonic emission and validated by experimental measurements with circularly polarized pump radiation - a condition that normally precludes SHG. Hence we identify the precise mechanism for second-harmonic emission in this macroscopically isotropic but microscopically ordered system, resolving confusion over whether the signal should be considered coherent SHG or incoherent hyperRayleigh scattering.

PACS number(s): 42.65.Ky, 87.22.Bt, 82.70.Kj
\end{abstract}

Macroscopic anisotropy is generally regarded as an inviolable condition for the emergence of a strong second harmonic from laser-irradiated systems [1]. It is for this reason that the technique of second-harmonic generation (SHG) has become a widely used tool for studying the surfaces of liquids and other centric systems [2], as no second-harmonic signal can be produced from their bulk. This is a powerful symmetry rule that extends beyond the electric-dipole approximation, remaining valid when all higher multipoles are taken into account $[3,4]$. It is then of great interest that a number of recent experimental studies have shown strong second-harmonic signals emerging from randomly oriented suspensions of the purple membrane $[5,6]$. An understanding of this signal is important not only for its fundamental significance, but also because of recent interest in potential device applications of the purple membrane [7].

A theory, fully cast in terms of molecular properties, has recently been developed to deal with SHG from suspensions [8]. It will be shown that this theory identifies the SHG detected in purple membrane suspensions as an emission that is coherent within each membrane unit $[6,8]$ - a conclusion supported by detailed studies of the angular distribution as reported below. The theory also predicts emergence of a second-harmonic signal under the condition of circularly polarized input. It is this feature that proves to be the key for pinpointing the mechanism and verifying the theory, since harmonic generation is then normally precluded in isotropic media [9].

Our experimental observations of second-harmonic emission from purple membrane suspensions in water were made with both plane and circularly polarized pump radiation. The excitation source was a mode-locked and $Q$-switched $\mathrm{Nd}$ :YAG laser (where YAG denotes yttrium aluminum garnet) providing 20-ps pulses of 1064-nm radiation at $10 \mathrm{~Hz}$. The energy density at the sample was varied over the range $10-100 \mathrm{~mJ} \mathrm{~cm}^{-2}$. The 532-nm signal was isolated from the pump radiation by a bandpass filter and monochromator and detected after passage through a polarizer. Polarization control was by a carefully oriented quarter wave plate.

\footnotetext{
*Author to whom correspondence should be addressed.
}

To elucidate the results, we here present key elements of a general theory of harmonic emission, accommodating salient polarization and angular-momentum features, based on the rigorous principles of microscopic quantum electrodynamics [1]. For the generation of any $n$ harmonic in a system comprising $M$ particles, the harmonic signal intensity $I$ derives from the equation

$$
\begin{aligned}
I= & \left(\frac{c k^{\prime 4} g_{\omega}^{(n)}}{8 \pi^{2} \varepsilon_{0}}\right)\left(\frac{I_{\omega}}{2 c \varepsilon_{0}}\right)^{n} \\
& \times\left|\sum_{u}^{M}\left(\boldsymbol{\beta}_{u} \overline{\mathbf{e}}^{\prime} \mathbf{e}_{1} \mathbf{e}_{2} \cdots \mathbf{e}_{n}\right) \exp \left(i \Delta \mathbf{k} \cdot \mathbf{R}_{u}\right)\right|^{2} .
\end{aligned}
$$

Here $\omega$ is the frequency of the pump laser, $I_{\omega}$ its intensity (power per unit cross section), and $g_{\omega}^{(n)}$ its degree of $n$ thorder coherence: $\boldsymbol{\beta}_{u}$ is the microscopic $n$ th-order unit polarizability, taken in tensor product with $\mathbf{e}$ and $\mathbf{e}^{\prime}$, the pump (fundamental) and harmonic polarization vectors, respectively, and the exponential term contains the wave-vector mismatch $\Delta \mathbf{k}=n \mathbf{k}-\mathbf{k}$. Both the $n$ th-order polarizability and the polarization vectors are assumed to be complex, to accommodate near-resonance behavior and circular polarizations, respectively; complex conjugates are denoted with an overbar. The position vector $\mathbf{R}_{u}$ is defined relative to the origin of an arbitrary Cartesian frame of reference having a fixed orientation within particle $u$.

For an isotropic system of units, as in a homogeneous fluid or suspension, we must take a rotational average for the ensemble response, as denoted below by angular brackets. The incoherent and coherent terms in Eq. (1) thus separate as diagonal (single center) and off-diagonal (two-center) terms, respectively:

$$
\begin{aligned}
I= & \left(\frac{c k^{\prime 4} g_{\omega}^{(n)}}{8 \pi^{2} \varepsilon_{0}}\right)\left(\frac{I_{\omega}}{2 c \varepsilon_{0}}\right)^{n}\left[\sum_{u}^{M}\left\langle\left|\left(\boldsymbol{\beta}_{u} \overline{\mathbf{e}}^{\prime} \mathbf{e}_{1} \mathbf{e}_{2} \cdots \mathbf{e}_{n}\right)\right|^{2}\right\rangle\right. \\
& +\sum_{u}^{M} \sum_{u^{\prime}}^{M-1}\left\langle\left(\boldsymbol{\beta}_{u} \overline{\mathbf{e}}^{\prime} \mathbf{e}_{1} \mathbf{e}_{2} \cdots \mathbf{e}_{n}\right)\right\rangle\left\langle\left(\overline{\boldsymbol{\beta}}_{u^{\prime}} \overline{\mathbf{e}} \mathbf{e}_{1} \mathbf{e}_{2} \cdots \mathbf{e}_{n}\right)\right\rangle \\
& \left.\times \exp \left(i \Delta \mathbf{k} \cdot \mathbf{R}_{u u^{\prime}}\right)\right]
\end{aligned}
$$


At this stage we note that the incoherent term encompasses a signal average and is linear in the number of units $M$, while the coherent term embodies a product of averages for the quantum amplitude of the interaction, scaling approximately as $M^{2}$. Each rotational average results in a quantity that is rotationally invariant and therefore necessarily scalar-a factor that plays a crucial role in the reasoning below [10]. It immediately follows that for isotropic systems, coherent emission of even $n$ harmonics is forbidden, as these entail a dependence on pump polarization that leads to a vector product of the polarization vector e with itself $[2,11]$.

For different reasons, the coherent generation of optical harmonics of any order (even or odd) with a circularly polarized pump is also generally forbidden within isotropic media [1]. This is one specific instance of an embargo for isotropic systems on any optical parametric process involving the absorption or emission of two or more circularly polarized photons of the same handedness and direction of propagation: the principle extends even to six-wave mixing and optically induced field mechanisms for second-harmonic generation [12]. The origin of this forbidden character supersedes the specific exclusion of even harmonics in isotropic media and is the basis for a number of recent studies on chiral surfaces [13-15]. It can be understood in several ways; the simplest is from angular momentum considerations.

It is evident that the annihilation of $n$ circularly polarized photons of a given handedness, coupled with the collinear emission of one $n$-harmonic photon, entails an exchange of at least $(n-1)$ units of angular momentum, precluding the necessarily scalar $(j=0)$ character for the quantum amplitude of the interaction. No such embargo applies for incoherent harmonic emission, principally because the $j=0$ rule there applies to signal intensity rather than amplitude. Since the intensity results from a coupling of the quantum amplitude with its complex conjugate, there is always a nonvanishing component with net angular momentum zero, under any polarization conditions. Therefore the coherent generation of a second harmonic from a circular pump is doubly forbidden; see also Ref. [16].

The coherent second-harmonic generation that we observe experimentally from purple membrane suspension is sustained, over a wide range of emission directions, when pumped with a circularly polarized fundamental and with a signal intensity larger by a factor of $\sim 1.3$ than with plane polarized pumping. This verifies that the signal results from the leading, diagonal term of Eq. (2), the detailed results for which is given as Eq. (26) in a previous paper [8]. It is now shown that the emission nonetheless has coherence attributes, which makes it impossible to regard simply as an incoherent (hyper-Rayleigh) second harmonic.

The purple membranes under consideration are isotropic in the bulk suspension, but each individual membrane contains many bacteriorhodospin (BR) molecules, each comprising the light-activated chromophores (retinal) responsible for the harmonic emission. It is well known that within the membrane unit these molecules have a structurally imposed orientational correlation, as confirmed by x-ray scattering [17] and electronic microscopy [18]. Specifically, it is observed that the BR transmembrane proteins, each of which contains a retinal chromophore, are arranged in a trimeric

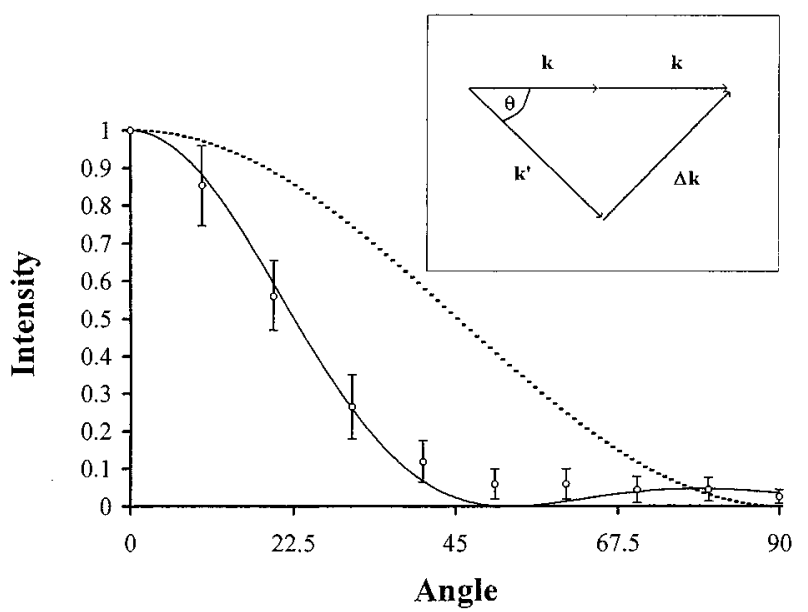

FIG. 1. Intensity (arbitrary units) of 532-nm optical second harmonic from a sample of purple membrane suspension, pumped with a plane-polarized $1.064-\mu \mathrm{m}$ fundamental, plotted against the angle of detection $\theta$ (in degrees). The theoretical result Eq. (9) is represented by the solid line, experimental data by the circles, and a $\cos ^{2}$ plot by the dashed line: error bars reflect the reproducibility of these measurements. The inset shows the phase matching (wave vectors are not to scale).

structure. Furthermore, these trimeric units form, in the membrane, an extended hexagonal array [19].

The second-harmonic emission from the isotropic suspension is explicitly given by the following equation, derived from the first term in the square brackets of Eq. (2) and expressed in a form that identifies the collective response of each microscopically ordered membrane unit labeled $u$ :

$$
I=M \frac{k^{\prime 4} I_{\omega}^{2} g_{\omega}^{(2)}}{32 \pi^{2} c \varepsilon_{0}^{3}}\left\langle\left|\boldsymbol{\beta}_{u} \overline{\mathbf{e} e \mathbf{e}}\right|^{2}\right\rangle .
$$

Here $\beta_{u}$ specifically represents the sum of all the individual retinal hyperpolarizabilities within a single membrane unit, each with its own relative position and orientation referred to a common Cartesian frame, such that

$$
\boldsymbol{\beta}_{u}=\sum_{\xi}^{N} \boldsymbol{\beta}_{\xi} \exp \left[i \Delta \mathbf{k} \cdots\left(\mathbf{R}_{\xi}-\mathbf{R}_{u}\right)\right] .
$$

Thus Eq. (3) can be rewritten as a sum of retinal pair contributions as

$$
I=M \frac{k^{\prime 4} I_{\omega}^{2} g_{\omega}^{2}}{32 \pi^{2} c \varepsilon_{0}^{3}} \sum_{\xi}^{N} \sum_{\xi^{\prime}}^{N-1}\left\langle\left(\boldsymbol{\beta}_{\xi} \overline{\mathbf{e}}^{\prime} \mathbf{e e}\right)\left(\overline{\boldsymbol{\beta}}_{\xi} \mathbf{e}^{\prime} \overline{\mathbf{e}} \overline{\mathbf{e}}\right) \exp \left(i \Delta \mathbf{k} \cdot \mathbf{R}_{\xi \xi^{\prime}}\right)\right\rangle
$$

in which both the retinal hyperpolarizabilities and also the vector $\mathbf{R}_{\xi \xi^{\prime}}$ have fixed mutual orientation. Here the rotational average must be effected with regard to the orientations of these quantities relative not only to the laboratory-fixed radiation tensor $\overline{\mathbf{e}}$ 'ee but also the phase factor $\Delta \mathbf{k}$. Using the cosine rule, the magnitude of $\Delta \mathbf{k}$ can be calculated in terms of the angle $\theta$ between the pump and harmonic radiation vectors (see the inset in Fig. 1) to give 


$$
|\Delta \mathbf{k}|^{2}=\frac{4 \omega^{2}}{c^{2}}\left(n_{\omega}^{2}+n_{\omega^{\prime}}^{2}-2 n_{\omega} n_{\omega^{\prime}} \cos \theta\right),
$$

where $n_{\omega}$ and $n_{\omega^{\prime}}$ are the refractive indices at the fundamental and harmonic frequencies, respectively. Equation (6) leads to two special cases. Maximum harmonic emission clearly occurs for forward emission, where $\cos \theta=1$, and the pump and harmonic waves propagate collinearly, giving

$$
|\Delta \mathbf{k}|=\left|2 \mathbf{k}-\mathbf{k}^{\prime}\right|=(2 \omega / c)\left(n_{\omega}-n_{\omega^{\prime}}\right) .
$$

This is the origin of the essentially directed character of the harmonic emission. For off-forward emission we can approximate the result (6) as

$$
|\Delta \mathbf{k}| \cong \frac{4 \omega}{c} \sqrt{n_{\omega} n_{\omega^{\prime}}} \sin \frac{\theta}{2} .
$$

For off-forward emission we proceed further by assuming that $\exp \left(i \Delta \mathbf{k} \cdot \mathbf{R}_{\xi \xi^{\prime}}\right)$ is a slowly varying function of $\mathbf{R}_{\xi \xi^{\prime}}$, defining a mismatch direction $z$ (not, in general, the forward direction) such that $\Delta \mathbf{k} \cdot \mathbf{R}_{\xi \xi^{\prime}}=|\Delta \mathbf{k}| z_{\xi \xi^{\prime}}$, with $z_{\xi \xi^{\prime}}$ denoting a component of the intermolecular displacement vector along the $z$ axis. Converting the sum in Eq. (5) to an integral between the limits of 0 and $L$, corresponding to the boundaries of the nonlinear material (here determined by the membrane perimeter), yields a sinc ${ }^{2}$ function, where $\operatorname{sinc} x=\sin x / x$. Thus for off-forward emission we find the harmonic function maps theoretically as

$$
I \sim \frac{N^{2} \sin ^{2}\left\{(4 \pi L / \lambda) \sqrt{n_{\omega} n_{\omega^{\prime}}} \sin (\theta / 2)\right\}}{\left\{(4 \pi L / \lambda) \sqrt{n_{\omega^{n} n^{\prime}}} \sin (\theta / 2)\right\}^{2}},
$$

where $\lambda$ is the pump laser wavelength and $N$ the number of retinal molecules per membrane unit $u$.

We now test our theoretical function against experimental data. Using the Nd:YAG wavelength of $1.064 \mu \mathrm{m}$, the effective length of the nonlinear membrane in suspension as 0.4 $\mu \mathrm{m}$ and the product of the refractive indices for the fundamental and harmonic frequencies in the suspension to be $\sim 2.25$, we have $7.09 \sin (\theta / 2)$ as the argument of the $\operatorname{sinc}^{2}$ function of Eq. (9). The experimental results were obtained for values of $\theta$ between zero and $90^{\circ}$. Plots of the experimental results and the $\operatorname{sinc}^{2}$ function as shown in Fig. 1, using the parameters outlined above, give an exceptional match, verifying the directed coherence of the harmonic signal from our isotropic suspension. Our equation also precisely fits the data obtained by Schmidt and Rayfield [6], who observed the lack of congruence with a simple $\cos ^{2} \theta$ fit (also shown in Fig. 1). Hence the results properly reflect a response that is coherent, in the sense that it arises from constructive interference between signals from various reti- nal molecules within each unit. The result nonetheless scales linearly with $M$, the number of such membrane units, given that coherence is sustained within each unit.

The individual retinal susceptibilities are, according to Eq. (5), essentially additive within the region over which coherence is sustained. Generally this may be regarded as the range of distances that satisfy the condition $\left|R_{\xi \xi^{\prime}}\right| \leqslant l_{c}$, where $l_{c}$ is the coherence length given by $\pi /|\Delta \mathbf{k}|$. As reported earlier [8], it may be supposed that for forward emission according to Eq. (7), the coherence length for the purple membrane in aquo lies somewhere between 25 and $50 \mu \mathrm{m}$, substantially in excess of the membrane dimensions. For nonforward scattering the coherence length exceeds the size of the individual membrane units at angles less than $\sim 25^{\circ}$; for angles greater than $25^{\circ}$ the coherence length is of similar magnitude to the estimated length of the membranes in question. It may be noted that, although one might conjecture an imposition of directionality on the harmonic emission not just by wave-vector matching but also by stimulated emission of the harmonic, the observed quadratic dependence of the harmonic signal on the pump intensity rules this out.

A final comment relates to two significant features of the polarization dependence: (a) the insensitivity to the orientation of a resolving polarizer of the harmonic produced by a plane polarized pump and (b) the approximately 1.3 increase in the forward-directed harmonic intensity produced by a circularly polarized pump. While these two features are not sufficient per se for characterization of all the parameters appearing in the salient rate equations-(21), (22), and (26) of Ref. [8] - it is readily apparent that together they are inconsistent with any simplification that might be conjectured on the assumption of Kleinman symmetry for the hyperpolarizability of bacteriorhodospin [20]. Physically, this signifies that the nonlinear response is dominated by resonance contributions, as suggested elsewhere [21-23].

In conclusion, we have observed coherent secondharmonic generation, in a macroscopically centrosymmetric system, with circularly polarized pump radiation. This measurement of such a doubly forbidden harmonic is fully accounted for by our theory, which pinpoints a precise interplay between the coherent and incoherent emission mechanisms at work. The angular distribution of the second harmonic in suspensions of bacteriorhodospin purple membrane unequivocally identifies coherent evolution of the harmonic within each membrane unit. The dependence on pump polarization is explicable only in terms of incoherent addition of harmonic signals from the different membrane units.

We thank Dr. P. J. Booth of Imperial College for preparation of the purple membrane samples. This work was supported by equipment grants from the Engineering and Physical Sciences Research Council (EPSRC) to S.R.M. and D.L.A. and by financial assistance from the EPSRC to P.A. and by the European Social Fund to A.J.W.
[1] D. L. Andrews, in Modern Nonlinear Optics, Part 2, edited by M. Evans and S. Kielich (Wiley, New York, 1993), p. 545.

[2] Y. R. Shen, Nature 337, 519 (1989).

[3] D. L. Andrews, J. Phys. B 13, 4091 (1980).

[4] D. L. Andrews and N. P. Blake, Phys. Rev. A 38, 3113 (1988).
[5] Q. Song, C. Wan, and C. K. Johnson, J. Phys. Chem. 98, 1999 (1994).

[6] K. Schmidt and G. W. Rayfield, Appl. Opt. 33, 4286 (1994).

[7] K. Clays, E. Hendrickx, M. Triest, T. Verbiest, A. Persoons, C. Dehu, and J.-L. Brédas, Science 262, 1419 (1993). 
[8] D. L. Andrews, P. Allcock, and A. A. Demidov, Chem. Phys. 190, 1 (1995).

[9] C. L. Tang and H. Rabin, Phys. Rev. B 3, 4025 (1971).

[10] The first and second sets of angular brackets in Eq. (2) are effected with respect to the orientation of a unit $u$ and the third with respect to the orientation of $u^{\prime}$, where $u$ and $u^{\prime}$ are orientationally uncorrelated.

[11] D. L. Andrews, J. Mod. Opt. 40, 939 (1993).

[12] D. L. Andrews, Nonlin. Opt. 8, 25 (1994).

[13] T. Petralli-Mallow, T. M. Wong, J. D. Byers, H. I. Yee, and J. M. Hicks, J. Phys. Chem. 97, 1383 (1993).

[14] J. D. Byers, H. I. Yee, and J. M. Hicks, J. Chem. Phys. 101, 6233 (1994).

[15] J. D. Byers, H. I. Yee, T. Petralli-Mallow, and J. M. Hicks, Phys. Rev. B 49, 14643 (1994).

[16] G. E. Stedman, in Modern Nonlinear Optics, Part 2 (Ref. [1]), p. 489.
[17] K. Hiraki, T. Hamanaka, T. Mitsui, and Y. Kito, Biochim. Biophys. Acta 647, 18 (1981).

[18] R. Henderson, J. M. Baldwin, T. A. Ceska, F. Zemlin, E. Beckmann, and K. H. Downing, J. Mol. Biol. 213, 899 (1990).

[19] Y. Shen, C. R. Safinya, K. S. Liang, A. F. Ruppert, and K. J. Rothschild, Nature 366, 48 (1993).

[20] Specifically, under the assumption of Kleinman index symmetry, $\beta_{(u) \lambda \lambda \mu} \beta_{(u) \mu \nu \nu}^{*}, \beta_{(u) \lambda \lambda \mu} \beta_{(u) \nu \nu \mu}^{*}, \beta_{(u) \lambda \mu \mu} \beta_{(u) \lambda \nu \nu}^{*}$, and $\beta_{(u) \lambda \mu \mu} \beta_{(u) \nu \nu \lambda}^{*}$ become equal, as do $\beta_{(u) \lambda \mu \nu} \beta_{(u) \lambda \mu \nu}^{*}$ and $\beta_{(u) \lambda \mu \nu} \beta_{(u) \mu \nu \lambda}^{*}$.

[21] Z. Chen, M. Sheves, A. Lewis, and O. Bouevitch, Biophys. J. 67, 1155 (1994).

[22] O. Bouevitch, A. Lewis, and M. Sheves, J. Phys. Chem. 99, 10648 (1995).

[23] E. Hendrickx, K. Clays, A. Persoons, C. Dehu, and J.-L. Brédas, J. Am. Chem. Soc. 117, 3547 (1995). 